\title{
Stability of High-Order Perturbative Methods for the Computation of Dirichlet-Neumann Operators
}

\author{
David P. Nicholls and Fernando Reitich \\ School of Mathematics, University of Minnesota, Minneapolis, Minnesota 55455 \\ E-mail: nicholls@math.umn.edu; reitich@math.umn.edu
}

Received June 28, 2000; revised February 2, 2001

In this paper we present results on the stability of perturbation methods for the evaluation of Dirichlet-Neumann operators (DNO) defined on domains that are viewed as complex deformations of a basic, simple geometry. In such cases, geometric perturbation methods, based on variations of the spatial domains of definition, have long been recognized to constitute efficient and accurate means for the approximation of DNO and, in fact, several numerical implementations have been previously proposed. Inspired by our recent analytical work, here we demonstrate that the convergence of these algorithms is, quite generally, limited by numerical instability. Indeed, we show that these standard perturbative methods for the calculation of DNO suffer from significant ill-conditioning which is manifest even for very smooth boundaries, and whose severity increases with boundary roughness. Moreover, and again motivated by our previous work, we introduce an alternative perturbative approach that we show to be numerically stable. This approach can be interpreted as a reformulation of classical perturbative algorithms (in suitable independent variables), and thus it allows for both direct comparison and the possibility of analytic continuation of the perturbation series. It can also be related to classical (preconditioned) spectral approaches and, as such, it retains, in finite arithmetic, the spectral convergence properties of classical perturbative methods, albeit at a higher computational cost (as it does not take advantage of possible dimensional reductions). Still, as we show, an alternative approach such as the one we propose may be mandated in cases where substantial information is contained in high-order harmonics and/or perturbation coefficients of the solution. (c) 2001 Academic Press

Key Words: Dirichlet-Neumann operators; geometric perturbation methods; numerical stability. 


\section{INTRODUCTION}

Classical models of mathematical physics typically involve equations for volumetric field quantities supplemented by relations on their restrictions and those of their normal derivatives at boundaries and interfaces. In many instances, moreover, the governing differential equations in "bulk" are simple (e.g., linear, homogeneous) and the analytical and numerical difficulties related to the model actually arise from geometrical complexity (e.g., irregular or unbounded domains) or from interfacial nonlinearities (as in classical moving boundary problems). In such cases, the dimensionality of the problem can conveniently be reduced by formulating it entirely in terms of surface quantities, provided normal derivatives can be related to these quantities. These relations, in turn, can be realized by simply introducing the notion of a Dirichlet-Neumann operator (DNO), and its higher order analogues, associated with the governing differential operator, and which is defined precisely so as to produce normal derivatives from boundary values. In this manner, the DNO has been brought to bear on problems (direct and inverse) relating to a wide variety of applications that include electromagnetic and acoustic scattering, nondestructive evaluation, and boundary value and free boundary problems from solid and fluid mechanics; see $(13,21),(16,28)$, and $(9,12)$, respectively, and the references therein. Within this framework then, a successful treatment of the corresponding models hinges on a thorough understanding of the mathematical properties of DNO and on the design of accurate and efficient numerical algorithms for their evaluation; these issues are the subject of the present discussion.

Among the myriad of methods that can be envisioned to evaluate DNO, methods based on boundary perturbations constitute an appealing alternative. These algorithms are based on the derivation of (low- or high-order) series representing the DNO in powers of a parameter measuring deviations from a separable, easily solvable geometry (e.g., planar, spherical, ellipsoidal) for which the DNO can be found explicitly. As has been demonstrated (9, 11, 21, 27), perturbation methods can lead very efficiently to accurate results within their domain of applicability. More importantly perhaps, and in contrast with alternative methods (e.g., finite elements or surface potentials), the implementation and performance of perturbative approaches do not depend strongly on the spatial dimension, a feature that makes them particularly attractive for three-dimensional calculations.

In fact, different versions of such schemes have been used in a variety of contexts; see e.g., $(9,11,17-24,27,29)$ and the references therein. Interestingly, however, rigorous justification and numerical analysis of these methods have been lacking, resulting in unclear domains of validity and rather arbitrary implementations; see, e.g., (11). Here we show that the main factor limiting the convergence of perturbative approaches of this type is the ill-conditioning inherent in the algorithmic formulation. We demonstrate that instabilities arise even when dealing with very smooth boundaries, and that their severity increases with boundary roughness. Finally, we show that an alternative perturbative method can be devised which is numerically stable, albeit with a somewhat higher computational cost than that associated with the classical (unstable) methods.

The examples and procedures we present were motivated by our recent development of a theoretical framework designed to shed light on the subtle issues underlying the justification of classical boundary variation algorithms (26). Specifically, we considered two main implementations of these methods which include the well-known Operator Expansion (OE) Method used by Milder et al. $(17,19-24,29)$ in the context of the boundary value problems of electromagnetic and acoustic scattering, and by a number of authors $(9,15,18,27,30)$ 
in the study of the classical free boundary model of gravity water waves (see Section 2). We also considered a different scheme, which we will refer to as the Field Expansion (FE) Method, that was proposed by Dommermuth and Yue (11), again in the context of water wave simulations, and that is based on the expansion of the full field quantities in bulk (see Section 2). For these implementations, our main findings of (26) relate to the realization that the corresponding algorithms rely mostly on cancellations to produce the perturbation series, and that these can be avoided with a reformulation of the problem in suitable independent variables. The possible numerical implications of these results, namely the potential for ill-conditioning and for a stabilized method, are rather evident and constitute the subject of this paper. For this, we first review in Section 2 the theoretical background and numerical implementation (i.e., the corresponding recursions) of the standard FE and OE methods. In particular, in Section 2.3, we provide both a detailed explanation and illustrative calculations of the nature of the underlying cancellations and resulting ill-conditioning. In Section 3 then, we describe our new perturbative method which, as we show with a variety of twoand three-dimensional numerical examples in Section 4, exhibits a very stable behavior and thus allows for a substantial enhancement in the accuracy and applicability of perturbative methods when compared to earlier approaches.

As we explain in Section 3, our new scheme can be related to classical (preconditioned) spectral algorithms. Our choice of a particular implementation that preserves the perturbative nature of the classical methods, leading to a series expansion, is two-fold. On one hand, it allows us to perform a direct comparison (of stability, domain of applicability, and so forth) with the more classical methods. On the other hand, it opens the possibility for incorporating analytic continuation mechanisms to accelerate and/or enhance the convergence of the series; see, e.g., (2, 3). The study of this latter possibility, and of its potential impact on a rather general setting of preconditioned spectral approximations (to accelerate the convergence of the underlying Neumann series), entails a further investigation of the analytic structure of the solution as a function of the perturbation parameter outside the disk of convergence of the series (see $(1,2)$ ), and will therefore be left for future work. Here we concentrate on the comparison with prior implementations - on stability, accuracy, and applicability. As we said, and in the spirit of spectral approximations in irregular domains, the method we propose does not take advantage of possible dimensional reductions, which are inherent in the OE and FE schemes and which result in their lower operation count. As follows from our discussion in Sections 3 and 4, this is due to the new "source terms" that appear in our recursion and which make a fully explicit evaluation of the higher order terms of the perturbation series difficult to achieve. This is in contrast with the homogeneous problems that arise in $\mathrm{OE}$ and FE methods and which admit relatively simple (closed-form) solutions. We expect however that a future study of the structure of the specific source terms in our recursion may suggest an accelerated scheme for the evaluation of the corresponding solutions (e.g., better adapted basis functions). In any case, and as we show, an alternative approach such as the one we introduce here may be mandated in cases where substantial information is contained in high-order harmonics and/or perturbation coefficients of the solution.

\section{PERTURBATIVE CALCULATION OF DNO}

As we said, our focus will be the numerical approximation of DNO through a (high-order) boundary perturbation scheme. Of course, the relevant DNO for a specific mathematical model will depend on the governing differential operators and the relevant geometrical 
arrangements. As will be evident, our results apply quite generally to perturbations of any exactly solvable problem. For definiteness, however, we shall concentrate on the DNO associated with Laplace's equation, and we shall work on a geometry motivated by classical problems in hydrodynamics $(9,11)$ where the basic unperturbed geometry is a "flat ocean"; the precise description follows in Section 2.1. There we also briefly review the theoretical background underlying boundary perturbation approaches, and in Section 2.2 we describe their numerical implementation as realized in the Operator Expansion (OE) and Field Expansion (FE) methods. The numerical instabilities associated with these are finally discussed in Section 2.3, leading the way to the introduction of our new algorithm in Section 3.

\subsection{Formulation and Theoretical Background}

To define the Dirichlet-Neumann operator, we consider a domain

$$
S_{\sigma}=\left\{(x, y) \in \mathbf{R}^{N-1} \times \mathbf{R} \mid-1<y<\sigma(x)\right\},
$$

and an arbitrary function $\xi$ ("the Dirichlet data") defined on $y=\sigma(x)$. For simplicity we shall assume that both $\sigma$ and $\xi$ are periodic with respect to a lattice $\Gamma$. However, the theoretical formalism that follows in this and subsequent sections can be easily adapted to deal with general (e.g., compactly supported) surfaces upon replacing Fourier series by Fourier transforms; see, e.g., (20) and the references therein. On the other hand, additional difficulties arise in this latter case that are associated with the implementation of numerical methods (specifically related to sampling and aliasing). Although a number of strategies, of varying degrees of sophistication and success, have been proposed to deal with these complications (see, e.g., $(6,17)$ ), we have chosen to work within a periodic context because our main conclusions should be largely independent of these details. Indeed, we expect that a similar discussion will apply in the case of nonperiodic surfaces, provided all relevant numerical methods are uniformly implemented following, for instance, the prescriptions in (6).

Associated with the domain $S_{\sigma}$, we shall thus define the DNO, $G(\sigma)$, as the operator

$$
G(\sigma) \xi=\partial_{\nu} v=\left.\nabla v\right|_{y=\sigma} \cdot\left(-\nabla_{x} \sigma, 1\right)^{T}
$$

where $v$ solves the (periodic) Dirichlet problem with boundary values $\xi$, that is

$$
\begin{array}{rlrl}
\Delta v(x, y) & =0 & & \text { in } S_{\sigma} \\
\partial_{y} v(x,-1) & =0 & & \\
v(x, \sigma(x)) & =\xi(x) & \\
v(x+\gamma, y) & =v(x, y) \quad \text { for all } \gamma \in \Gamma .
\end{array}
$$

We note that the DNO can also be defined for a domain of infinite extent by replacing Eq. (3b) with the condition

$$
\partial_{y} v(x, y) \rightarrow 0 \quad \text { as } y \rightarrow-\infty
$$

The accurate numerical evaluation of DNO in irregular domains is evidently a nontrivial matter as it entails, directly or indirectly, the approximation of (nonconvolution) singular 
integrals; see, e.g., (10). There is, however, one exception: For a separable geometry, the operator (of convolution-type in this very special case) can be explicitly found. In our framework, such a geometry is provided by a "flat ocean," corresponding to $\sigma=0$. In this case, we have

$$
G(0) \xi=|D| \tanh (|D|) \xi(x)=\sum_{k \in \Gamma^{\prime}}|k| \tanh (|k|) \hat{\xi}(k),
$$

where $D=-i \nabla_{x}$ and $\Gamma^{\prime}$ is the conjugate lattice to $\Gamma$ (i.e., wavenumbers). In view of this, a perturbative approach is suggested whereby a general surface is viewed as a deviation from a plane. More precisely, a family of surfaces $\sigma=\varepsilon f,|\varepsilon| \leq \bar{\varepsilon}$, gives rise to DNO, $G(\varepsilon f)$, and a perturbation series

$$
G(\varepsilon f) \xi=\sum_{n=0}^{\infty}\left(G_{n}(f) \xi\right) \varepsilon^{n}
$$

could be used for their approximation. The feasibility of such an approach obviously hinges on two main factors: (1) the convergence of the series (6), and (2) the development of an algorithm for the efficient evaluation of its coefficients.

The question of convergence of the series (6) has a long history and, for two-dimensional domains (i.e., $N=2$ ), an affirmative answer can be derived from the work of Calderón (4) and Coifman and Meyer (7). Indeed, it follows from these that for any Lipschitz profile $f$ there exists a constant $B>0$ such that

$$
\left\|G_{n}(f) \xi\right\|_{L^{2}} \leq C\|\xi\|_{H^{1}} B^{n}
$$

which implies that the series (6) converges in $L^{2}$ for sufficiently small values of $\varepsilon$. Extensions of these results to higher dimensions were recently established by Craig, Schanz, and Sulem (10) and Craig and Nicholls (8); see also Nicholls and Reitich (26).

As for the numerical evaluation of the Taylor coefficients $G_{n}(f)$ in (6), the perturbative nature of the series implies that, at least formally, they can be recursively obtained. In the next section, we review two implementations of these recursions that have been previously proposed. As we explain in Section 2.3 (and further demonstrate numerically in Section (4)) these algorithms, though very efficient, are limited by their conditioning properties.

\subsection{The Field Expansion and Operator Expansion Methods}

A natural approach to the perturbative approximation of DNO, which we shall refer to as the Field Expansions (FE) Method, consists of simply expanding the field $v=v(x, y, \varepsilon)$ solving Eqs. (3a)-(3d) (or (3a), (3c), (3d), and (4)) in the form

$$
v(x, y, \varepsilon)=\sum_{n=0}^{\infty} v_{n}(x, y) \varepsilon^{n}
$$

and, a posteriori, of computing the DNO based on this expansion via the formula

$$
G_{n}(f) \xi=-\nabla_{x} f \cdot \sum_{l=0}^{n-1} \frac{f^{l}}{l !} \partial_{y}^{l} \nabla_{x} v_{n-1-l}(x, 0)+\sum_{l=0}^{n} \frac{f^{l}}{l !} \partial_{y}^{l+1} v_{n-1}(x, 0)
$$


(see (11)). For instance, in the case of infinite depth (cf. (4)) it is easy to show that the functions $v_{n}(x, y)$ must satisfy

$$
\begin{aligned}
\Delta v_{n}(x, y) & =0 & & \text { in } S_{0} \\
\partial_{y} v_{n}(x, y) & \rightarrow 0 & & \text { as } y \rightarrow-\infty \\
v_{n}(x, 0) & =H_{n}(x) & & \\
v_{n}(x+\gamma, y) & =v_{n}(x, y) & & \text { for all } \gamma \in \Gamma,
\end{aligned}
$$

where

$$
H_{n}(x)=-\sum_{l=0}^{n-1} \frac{f^{n-l}}{(n-l) !} \partial_{y}^{n-l} v_{l}(x, 0)+\delta_{n, 0} \xi(x),
$$

and $\delta_{j, k}$ is the Kronecker delta. A spectral representation of the solution of Eqs. (10a), (10b), and (10d) is given by

$$
v_{n}(x, y)=\sum_{k \in \Gamma^{\prime}} d_{n, k} e^{i k \cdot x+|k| y}
$$

where $d_{n, k}$ are Fourier coefficients. Equation (10c) then translates into the recursion

$$
d_{n, k}=-\sum_{l=0}^{n-1} \sum_{q \in \Gamma^{\prime}} C_{n-l, k-q} d_{l, q}|q|^{n-l}+\delta_{n, 0} \hat{\xi}(k),
$$

where the numbers $C_{l, k}$ are the Fourier coefficients of the function $f(x)^{l} / l$ !, that is

$$
\frac{f(x)^{l}}{l !}=\sum_{k \in \Gamma^{\prime}} C_{l, k} e^{i k \cdot x} .
$$

Equation (13) can be used to recursively evaluate the coefficients $d_{n, k}$ and these, in turn, allow for the calculation of the Fourier representation of $G_{n}(f)$ by means of Eq. (9).

An alternative and elegant scheme for the calculation of the operators $G_{n}(f)$ has been used by a number of authors in various applications, including the study of gravity water waves $(9,15,18,27,30)$ and ocean scattering $(17,19-24,29)$. The method works directly with the DNO without reference to the bulk potential and has thus been termed the Operator Expansion (OE) Method. To review this approach, let us assume again that the basic geometry is of infinite extent in $y$, in which case the unperturbed DNO is given by

$$
G(0) \xi=|D| \xi \equiv \sum_{k \in \Gamma^{\prime}}|k| \hat{\xi}(k)
$$

Since the function

$$
w_{p}(x, y)=e^{i p \cdot x+|p| y}
$$

is a solution of Eqs. (3a), (3d), and (4) we have

$$
G(\varepsilon f)\left[e^{i p x+|p| \varepsilon f}\right]=\left.\left(\partial_{y}-\varepsilon \nabla_{x} f \cdot \nabla_{x}\right)\left(e^{i p \cdot x+|p| y}\right)\right|_{y=\varepsilon f},
$$


that is

$$
G(\varepsilon f)\left[e^{i p x+|p| \varepsilon f}\right]=\left(|p|-\varepsilon \nabla_{x} f \cdot i p\right) e^{i p \cdot x+|p| \varepsilon f} .
$$

Thus, expanding the equality (18) in the form of a series in $\varepsilon$ and equating like powers, we obtain the recursion

$$
\begin{aligned}
G_{n}(f) e^{i p \cdot x}= & \frac{f^{n}}{n !}|p|^{n+1} e^{i p \cdot x}-\left(\nabla_{x} f\right) \frac{f^{n-1}}{(n-1) !} \cdot(i p)|p|^{n-1} e^{i p \cdot x} \\
& -\sum_{l=0}^{n-1} G_{l}(f)\left[\frac{f^{n-l}}{(n-l) !}|p|^{n-l} e^{i p \cdot x}\right]
\end{aligned}
$$

or, symbolically,

$$
G_{n}(f) \xi(x)=D \frac{f^{n}}{n !} D|D|^{n-1} \xi(x)-\sum_{l=0}^{n-1} G_{l}(f)\left[\frac{f^{n-l}}{(n-l) !}|D|^{n-l} \xi(x)\right] .
$$

Finally, using the self-adjoint nature of $G_{n}(f)$ and $|D|$, we may rewrite (20) in the form

$$
G_{n}(f) \xi(x)=|D|^{n-1} D \frac{f^{n}}{n !} D \xi(x)-\sum_{l=0}^{n-1}|D|^{n-l} \frac{f^{n-l}}{(n-l) !} G_{l}(f) \xi(x),
$$

which gives a direct recurrence for the operators $G_{n}(f)$.

\subsection{Cancellations and Ill-Conditioning}

It is important to note that the above derivations of (13) and (21) are formal in nature. Indeed, although the results in $(4,7,8,10,26)$ do guarantee the convergence of the expansion (6), the validity of these recursions demands more careful consideration. In fact, at first glance the formulas would seem to require a high degree of regularity on the profile $f$, as is most evidently displayed in Eq. (21). On the other hand, the theoretical results on analyticity of DNO apply to general "rough" (Lipschitz or $C^{1}$ ) perturbations of a plane. As conjectured in (26), this apparent contradiction is at the heart of the unstable behavior of the OE and FE algorithms in high-order calculations. Indeed, as argued there, substantial cancellations occur in (13) and (21) so that the overall sums in their respective right-hand sides give rise to finite quantities in spite of possible singularities in the individual terms.

In fact, substantial insight into the nature of these cancellations can be garnered by explicit consideration of the recursion (21) to second order. Indeed, it follows from (15) and (21) that

$$
\begin{aligned}
& G_{0} \xi=|D| \xi \\
& G_{1} \xi=D f D \xi-|D| f G_{0} \xi \\
& G_{2} \xi=|D| D \frac{f^{2}}{2} D \xi-|D|^{2} \frac{f^{2}}{2} G_{0} \xi-|D| f G_{1} \xi
\end{aligned}
$$

where $G_{j}=G_{j}(f)$. In particular, the definition of $G_{2} \xi$ appears to entail second derivatives of the profile $f$ and third derivatives of the Dirichlet data $\xi$. On the other hand, we know 
(cf. Eq. (7)) that $G_{2} \xi$ is guaranteed to be square integrable under the sole assumption that $f$ is Lipschitz and that $\xi$ is in $H^{1}$ (a single derivative is square integrable). As we shall now see, these apparently contradictory remarks can in fact be reconciled, as the most singular part of $G_{2} \xi$ can be shown to exactly cancel out. For this, we shall make use of a kind of "product rule" for $G_{0}=|D|$ that can be easily verified. More precisely, we shall use the fact that for any given functions $\phi$ and $\psi$, the function $G_{0}(\phi \psi)$ can be written as

$$
G_{0}(\phi \psi)=G_{0}(\phi) \psi+\phi G_{0}(\psi)+R
$$

where the "remainder" $R$ is more regular than each of the other terms. In the present periodic context, the higher regularity of $R$ can be readily verified by considering the decay of its Fourier coefficients.

Next, we note that $G_{2} \xi=|D| \eta$ where, using (22a) and (22b),

$$
\begin{aligned}
\eta & =D \frac{f^{2}}{2} D \xi-|D| \frac{f^{2}}{2} G_{0} \xi-f G_{1} \xi \\
& =D \frac{f^{2}}{2} D \xi-|D| \frac{f^{2}}{2}|D| \xi-f D f D \xi+f|D| f|D| \xi
\end{aligned}
$$

and we shall show that the most singular part, $\eta_{s}$, of $\eta$ (i.e., that involving first derivatives of $f$ and second derivatives of $\xi$ ) does in fact vanish. Indeed, using (23), and the equality $|D|^{2}=D^{2}$, we find

$$
\begin{aligned}
\eta_{s}= & {\left[f(D f)(D \xi)+\frac{f^{2}}{2} D^{2} \xi\right]-\left[f(|D| f)(|D| \xi)+\frac{f^{2}}{2}|D|^{2} \xi\right] } \\
& -\left[f(D f)(D \xi)+f^{2} D^{2} \xi\right]+\left[f(|D| f)(|D| \xi)+f^{2}|D|^{2} \xi\right] \\
= & 0
\end{aligned}
$$

as predicted.

The effect of this type of cancellations on the conditioning of the OE and FE methods shall be thoroughly exemplified in Section 4 through a variety of two- and three-dimensional numerical experiments, as we compare their performance to that of a new stable algorithm that we introduce in Section 3. Here, and to further motivate the need for a better conditioned approach, let us simply consider the case of smooth (one-dimensional) perturbations of the plane $y=0$ with

$$
f(x)=\cos (x)
$$

and let us take, for definiteness, the Dirichlet data

$$
\xi(x)=\cos (x)
$$

In this case, a calculation in exact (rational) arithmetic can be performed by resorting to a symbolic manipulator (Maple, in our case) with rather modest memory and time requirements. In this manner, the precise values of the Fourier coefficients $a_{n, k}$ of the periodic function

$$
G_{n}(f) \xi=\sum_{k \in \Gamma^{\prime}} a_{n, k} e^{i k \cdot x}
$$




\section{TABLE I}

Significant Digits in Real Part of $a_{n, 1}$;

Smooth Profile (2D)

\begin{tabular}{rrr}
\hline$n$ & FE & OE \\
\hline 2 & 16 & 16 \\
6 & 15 & 16 \\
10 & 13 & 15 \\
14 & 13 & 13 \\
18 & 10 & 12 \\
22 & 7 & 9 \\
26 & 5 & 6 \\
30 & 2 & 5 \\
34 & 0 & 1 \\
\hline
\end{tabular}

can be obtained and compared to the outcome of spectral implementations OE and FE in double precision arithmetic. The results of such an exercise are reported in Table I for the Fourier coefficient $a_{n, k}$ corresponding to wavenumber $k=1$; the behavior for other coefficients is qualitatively similar, and it deteriorates with increasing wavenumber. We see that even in this most favorable case of analytic, low-frequency perturbations and Dirichlet data, there is a substantial loss of accuracy in the calculation of the coefficients $a_{n, k}$ as $n$ increases: Approximately one digit is lost every time the number of derivatives $n$ increases by two beyond $n=6$. We remark that the OE and FE results were computed with 128 Fourier modes so that no aliasing errors are incurred; the loss of accuracy is solely due to ill-conditioning. As we show in Section 4, these instabilities become more pronounced for more irregular perturbations, and they may actually pose significant limitations on the applicability of these algorithms.

\section{A NEW NUMERICAL ALGORITHM}

The example above shows that the $\mathrm{OE}$ and $\mathrm{FE}$ approaches may be inappropriate for high-order calculations. On the other hand, in the context of perturbative methods, highorder approximations may be mandated by accuracy requirements, particularly for larger surface deformations. In this section we introduce an alternative algorithm that allows for the accurate evaluation of higher order terms in the perturbation series. The algorithm is inspired by our recent work (26), where we showed that a simple change of independent variables leads to a perturbative scheme that, in contrast with the OE and FE methods, does not rely on cancellations. This characteristic allowed us in (26) to use the corresponding recursion to provide an alternative proof of analyticity of DNO. And, moreover, it also suggested that a numerical implementation should result in a well-conditioned procedure, thus enabling high-order approximations. As we shall see (Section 4) this is indeed the case and, in fact, our new algorithm can be used to substantially enhance the applicability of geometric perturbation approaches.

As we said, the method we propose is a modification of the FE approach that includes a change of independent variables. This change of variables has the effect of transforming the perturbed domain onto the basic, unperturbed geometry, and we shall therefore refer to it as the method of Transformed Field Expansions (TFE). In particular, the transformation 
simplifies the geometry and thus makes it amenable to a spectral collocation technique; see, e.g., (5) and the references cited therein. As is well-known, however, a direct implementation of such generally leads to inefficient and poorly conditioned algorithms, and a variety of iterative and preconditioning mechanisms have been proposed; see, e.g., $(5,25)$. On the other hand, and as we describe below, our solution procedure for the transformed problem will be chosen so as to retain the perturbative nature on the size of the boundary variation. As we mentioned in Section 1, this allows for a direct comparison (of stability and applicability) with the OE and FE implementations, and it also provides a stable mechanism for the evaluation of a perturbation series whose structure could be further exploited by analytic continuation $(2,3)$. Moreover, the technique we propose is closely related to the aforementioned iterative preconditioned spectral methods and, in fact, it would reduce to a version of these if the transformed equation depended linearly on the size of the perturbation. To see this, consider the change of variables

$$
\begin{aligned}
& x^{\prime}=x \\
& y^{\prime}=\frac{y-\varepsilon f(x)}{1+\varepsilon f(x)},
\end{aligned}
$$

mapping the irregular domain $S_{\varepsilon f}$ onto the strip $S_{0}$. Then, the field

$$
u\left(x^{\prime}, y^{\prime}, \varepsilon\right)=v\left(x^{\prime}, y^{\prime}\left(1+\varepsilon f\left(x^{\prime}\right)\right)+\varepsilon f\left(x^{\prime}\right), \varepsilon\right)
$$

will satisfy (cf. Eq. (3a)-(3d))

$$
\begin{array}{rlrl}
\Delta^{\prime} u-\varepsilon Q(u) & =0 & & \text { in } S_{0} \\
\partial_{y^{\prime}} u(x,-1) & =0 & & \\
u\left(x^{\prime}, 0\right) & =\xi\left(x^{\prime}\right) & \\
u\left(x^{\prime}+\gamma, y^{\prime}\right) & =u\left(x^{\prime}, y^{\prime}\right) \quad \text { for all } \gamma \in \Gamma,
\end{array}
$$

where the operator $Q$ is given by

$$
\begin{aligned}
Q(u)= & \operatorname{div}_{x^{\prime}}\left[\frac{\left(\nabla_{x^{\prime}} f\right)\left(1+y^{\prime}\right)}{1+\varepsilon f} \partial_{y^{\prime}} u\right]+\partial_{y^{\prime}}\left[\frac{\left(1+y^{\prime}\right)}{1+\varepsilon f} \nabla_{x^{\prime}} f \cdot \nabla_{x^{\prime}} u-\frac{\varepsilon\left|\nabla_{x^{\prime}} f\right|^{2}\left(1+y^{\prime}\right)^{2}}{(1+\varepsilon f)^{2}} \partial_{y^{\prime}} u\right. \\
& \left.+\frac{2 f+\varepsilon f^{2}}{(1+\varepsilon f)^{2}} \partial_{y^{\prime}} u\right]-\frac{1}{1+\varepsilon f} \nabla_{x^{\prime}} f \cdot \nabla_{x^{\prime}} u+\frac{\varepsilon\left|\nabla_{x^{\prime}} f\right|^{2}\left(1+y^{\prime}\right)}{(1+\varepsilon f)^{2}} \partial_{y^{\prime}} u .
\end{aligned}
$$

Next, note that, since the transformation (25a) and (25b) coincides with the identity when $\varepsilon=0$, the coefficients $u_{n}$ in an expansion

$$
u\left(x^{\prime}, y^{\prime}, \varepsilon\right)=\sum_{n=0}^{\infty} u_{n}\left(x^{\prime}, y^{\prime}\right) \varepsilon^{n}
$$

can still be recursively obtained. In particular, if $Q$ were independent of $\varepsilon$, the recursion would simply read

$$
\Delta^{\prime} u_{n}=\varepsilon Q\left(u_{n-1}\right)
$$


with appropriate boundary conditions or, in terms of the partial sums $U^{n}=\sum_{k=0}^{n} u_{k} \varepsilon^{k}$,

$$
\Delta^{\prime} U^{n}=\varepsilon Q\left(U^{n-1}\right)
$$

since $Q$ depends linearly on $u$. This last equation can be equivalently written as

$$
\Delta^{\prime} U^{n}=\Delta^{\prime} U^{n-1}-\left[\Delta^{\prime} U^{n-1}-\varepsilon Q\left(U^{n-1}\right)\right],
$$

which coincides with a (Richardson) iteration scheme associated to the solution of $\Delta^{\prime} u-$ $\varepsilon Q(u)=0$ upon preconditioning with the Laplacian (5).

In the case of Eqs. (27a)-(27d), of course, $Q$ does depend on $\varepsilon$, so that a direct perturbative treatment leads to a somewhat more complicated recursion than that in (30). Indeed, in this case and upon dropping the primes, the recursion reads

$$
\begin{array}{ll}
\Delta u_{n}(x, y)=\left(1-\delta_{n, 0}\right) F_{n}(x, y) & \text { in } S_{0} \\
\partial_{y} u_{n}(x,-1)=0 & \\
u_{n}(x, 0)=\delta_{n, 0} \xi(x) & \text { for all } \gamma \in \Gamma, \\
u_{n}(x+\gamma, y)=u_{n}(x, y) &
\end{array}
$$

where

$$
F_{n}(x, y)=\operatorname{div}_{x}\left[F_{n}^{(1)}(x, y)\right]+\partial_{y} F_{n}^{(2)}(x, y)+F_{n}^{(3)}(x, y),
$$

and

$$
\begin{aligned}
F_{n}^{(1)}(x, y)= & (1+y)\left(\nabla_{x} f\right) \sum_{l=0}^{n-1}(-f)^{l} \partial_{y} u_{n-1-l} \\
F_{n}^{(2)}(x, y)= & (1+y)\left(\nabla_{x} f\right) \cdot \sum_{l=0}^{n-1}(-f)^{l} \nabla_{x} u_{n-1-l}-(1+y)^{2}\left|\nabla_{x} f\right|^{2} \\
& \times \sum_{l=0}^{n-2}(l+1)(-f)^{l} \partial_{y} u_{n-2-l}+f \sum_{l=0}^{n-1}(l+2)(-f)^{l} \partial_{y} u_{n-1-l} \\
F_{n}^{(3)}(x, y)= & -\left(\nabla_{x} f\right) \cdot \sum_{l=0}^{n-1}(-f)^{l} \nabla_{x} u_{n-1-l}+(1+y)\left|\nabla_{x} f\right|^{2} \\
& \times \sum_{l=0}^{n-2}(l+1)(-f)^{l} \partial_{y} u_{n-2-l}
\end{aligned}
$$

(see (26)). The TFE approach will therefore be based on the solution of this recurrence and on the relation

$$
G(\varepsilon f) \xi=-\varepsilon \nabla_{x} f \cdot \nabla_{x} u(x, 0, \varepsilon)+\frac{1+\varepsilon^{2}\left|\nabla_{x} f\right|^{2}}{1+\varepsilon f} \partial_{y} u(x, 0, \varepsilon),
$$


satisfied by the DNO in the new variables (primes dropped), from which a new formula can be derived for the $n$-th term in its Taylor series expansion

$$
\begin{aligned}
G_{n}(f) \xi= & \sum_{l=0}^{n}(-f)^{l} \partial_{y} u_{n-l}(x, 0)-\delta_{n, 1} \nabla_{x} f \cdot \nabla_{x} \xi(x) \\
& +\left|\nabla_{x} f\right|^{2} \sum_{l=0}^{n-2}(-f)^{l} \partial_{y} u_{n-2-l}(x, 0) .
\end{aligned}
$$

As was shown in (26), the formulation above allows for the direct recursive estimation of the functions $u_{n}$ (in appropriate Sobolev norms) indicating that, in contrast with (13) and (21), this new recurrence does not entail significant cancellations. In the next section, we present a variety of two- and three-dimensional numerical examples that clearly show the beneficial effect that this has on the stability, accuracy, and applicability of the method as we compare its performance to that of the $\mathrm{OE}$ and $\mathrm{FE}$ implementations.

\section{NUMERICAL IMPLEMENTATION AND RESULTS}

In order to substantiate the theoretical predictions that suggested the unstable behavior of $\mathrm{OE}$ and FE and the improved numerical properties of TFE, we have conducted a thorough study of the three algorithms in their two- and three-dimensional versions. Here we report on the implementation details and on the outcome of this comparison, showing that indeed instabilities invariably arise in $\mathrm{OE}$ and FE implementations and that substantial precision can be gained from use of TFE in high-order approximations.

\subsection{Numerical Implementation}

The implementation of OE is based upon the evaluation of Eq. (21) (or its analogue in finite depth). The periodic boundary conditions in the $x$ variable and the conspicuous appearance of Fourier multipliers in the formula naturally suggest a Fourier spectral method. In this scheme, the unknowns are represented by Fourier series of a fixed order $N_{x} / 2$ (with $N_{x}$ collocation points), and all products are evaluated using fast (de-aliased) convolutions via the FFT algorithm.

The FE approximation, on the other hand, is constructed from the recurrence (13) (or its analogue in finite depth) and the representation (9). Once again, a Fourier basis is very natural, and we thus use a Fourier spectral approach with fast convolutions.

The implementation of the TFE approximation is somewhat different from that of the OE and FE methods. Indeed, both the $\mathrm{OE}$ and FE approaches rely on the homogeneity of the differential equation (10a) to express the solutions in closed form as linear combinations of suitable basis functions (exponentials in this case, cf. (12), (16)). In contrast, the "source terms" in (31a) preclude the use of a standard basis for the exact representation of solutions, which we therefore approximate numerically. As we said, the consequent increase in computational cost may be compensated by a substantial increase in accuracy that can, in fact, allow for computations beyond the reach of the OE and FE algorithms (see Section 4.3).

For the numerical solution of (31a)-(31d) we have chosen a spectral FourierChebyshev tau method which posits an approximate solution of the form

$$
\tilde{u}_{n}(x, y)=\sum_{k \in \Gamma^{\prime},|k|<N_{x} / 2} \sum_{l=0}^{N_{y}} \hat{u}_{n}(k, l) e^{i k \cdot x} T_{l}(2 y+1),
$$


where $T_{l}(z)$ is the $l$-th Chebyshev polynomial. The resulting set of equations can be efficiently solved via the use of fast Fourier and Chebyshev transforms in conjunction with the fast elliptic solve outlined in (14, Section 10). Finally, the DNO is approximated from this representation through Eq. (35).

\subsection{Convergence and Conditioning Tests}

A standard approach to the measurement of convergence properties of a numerical method is by comparison to an easily evaluated exact solution. In the context of DNO, such solutions can be produced for any surface profile $f(x)$ by considering the particular solutions to Eqs. (3a), (3b), and (3d) given by

$$
v_{k}(x, y)=\cosh (|k|(y+1)) \cos (k \cdot x) .
$$

For these, we obviously have

$$
G(f)\left(v_{k}(x, f(x))\right)=\left.\nabla v_{k}\right|_{y=f} \cdot\left(-\nabla_{x} f, 1\right)^{T}
$$

for any function $f$. Then, for the outcome of a numerical simulation, the defect in this relation (e.g., in the discrete $L^{2}$ norm) can be used as an error estimate. As we show below, the results of such experiments are directly relevant to the determination of the accuracy and applicability of the different methods. However, they provide only indirect evidence as to the ill-conditioning of the $\mathrm{OE}$ and FE schemes.

To garner more immediate insight into the nature and effects of these instabilities, we shall also present the results of a different set of tests. In these tests, a direct comparison is made of the values of the coefficients $a_{n, k}$ (cf. (24)) in the Fourier series of the DNO applied to a particular Dirichlet datum (see Section 2.3). To compute the "exact" values of these coefficients, we resort to a symbolic manipulator (e.g., Maple) with rational arithmetic or, when such calculations exceed memory or time constraints, to quadruple precision simulations. In this manner, a clear picture emerges of the degradation of the numerical results as the approximation order is increased.

\subsection{Numerical Results}

We have performed numerical convergence and conditioning tests on the TFE, FE, and OE methods using several sample profiles in two and three dimensions. For the sake of definiteness, all computations were performed on domains of finite extent in $y$ corresponding to Eqs. (3a)-(3d).

4.3.1. Two-dimensional computations. The discussions above indicate that, overall, the performance of TFE should improve over that of FE and OE, and that this improvement should be enhanced for rougher boundaries. To confirm this predicted behavior we have selected two-dimensional domains whose upper boundaries are shaped by the profiles

$$
\begin{aligned}
& f_{s}(x)=\cos (x) \\
& f_{r}(x)=\left(2 \times 10^{-4}\right) x^{4}(2 \pi-x)^{4}-c_{0} \\
& f_{L}(x)= \begin{cases}-\frac{2}{\pi} x+1 & 0 \leq x \leq \pi \\
\frac{2}{\pi} x-3 & \pi \leq x \leq 2 \pi\end{cases}
\end{aligned}
$$


intended to be prototypical of "smooth," "rough" (finite smoothness, $C^{4}$ ), and Lipschitz boundaries, respectively. The constant $c_{0}$ in (39b) was chosen so that $f_{r}$ has zero mean (as do $f_{s}$ and $f_{L}$ ). To facilitate a comparison, the scaling of each of the profiles was chosen so that they all have approximate amplitudes of 2 , and maximum slopes of about 1 . The Fourier series representation of $f_{r}$ and $f_{L}$ are given by

$$
\begin{aligned}
& f_{r}(x)=\sum_{k=1}^{\infty} \frac{96\left(2 k^{2} \pi^{2}-21\right)}{125 k^{8}} \cos (k x) \\
& f_{L}(x)=\sum_{k=1}^{\infty} \frac{8}{\pi^{2}(2 k-1)^{2}} \cos ((2 k-1) x),
\end{aligned}
$$

and in order to minimize the effects of aliasing errors, we approximate them by their truncated Fourier series

$$
\begin{aligned}
& f_{r, P}(x)=\sum_{k=1}^{P} \frac{96\left(2 k^{2} \pi^{2}-21\right)}{125 k^{8}} \cos (k x) \\
& f_{L, P}(x)=\sum_{k=1}^{P / 2} \frac{8}{\pi^{2}(2 k-1)^{2}} \cos ((2 k-1) x) .
\end{aligned}
$$

If $P \ll N_{x} / 2$, the number of modes in our numerical approximation, the effects of aliasing will be minimal (in fact, no aliasing occurs if $n P+F \leq N_{x} / 2$ where $n$ is the degree of the perturbation series and $F$ is the number of Fourier modes in the Dirichlet data).

In the first set of experiments, we explore the conditioning of the methods as described in Section 4.2. via computations of the Fourier coefficients $a_{n, k}$ (see (24)) corresponding to each of the profiles $f_{s}, f_{r, P}$, and $f_{L, P}(P=40)$, and for the fixed Dirichlet datum

$$
\xi(x)=\cos (x)
$$

$(F=1)$. This choice of $\xi$ is taken so as to simplify the "exact" calculation of the $a_{n, k}$, which was performed in rational arithmetic in the case of $f_{s}$ and in quadruple precision for $f_{r, P}$ and $f_{L, P}$. We have measured the outcome of these comparisons by the number of "digits of accuracy" which, for an approximation $p$ to an exact value $p^{*}$, is defined to be the largest integer $t$ such that

$$
\frac{\left|p-p^{*}\right|}{|p|}<5 \times 10^{-t} .
$$

The results of these experiments for the real part of $a_{n, 1}$ are given in Tables II-IV; the behavior for other modes is qualitatively similar and deteriorates with increasing wavenumber. In each case, the choice of $N_{x}$ was taken so as to optimize the results of OE and FE: sufficiently large to avoid significant aliasing but small enough so as to minimize the effects of numerical ill-conditioning. As a result we observe in Table II for instance, that while, as discussed in Section 2.3 (Table I), the accuracy of the OE and FE methods degenerates at a rate of one digit per two terms beyond $n=6$, that of TFE remains virtually constant throughout the range of orders; the precise value of this constant is, of course, determined by our resolution of the vertical dependence of the field ( $N_{y}=64$ in all three cases). The results in Tables III 
TABLE II

Significant Digits in Real Part of $a_{n, 1}$; Smooth Profile (2D, $N_{x}=128$ )

\begin{tabular}{rrrr}
\hline$n$ & TFE & FE & OE \\
\hline 2 & 11 & 16 & 16 \\
6 & 10 & 15 & 16 \\
10 & 10 & 13 & 15 \\
14 & 10 & 13 & 13 \\
18 & 9 & 10 & 12 \\
22 & 9 & 7 & 9 \\
26 & 9 & 5 & 6 \\
30 & 9 & 2 & 1 \\
34 & 9 & 0 & 5 \\
\hline
\end{tabular}

and IV are similar, with the $\mathrm{OE}$ and FE results deteriorating at a faster rate as the profile becomes less regular.

Next, we compare the convergence behavior of the three methods in this two-dimensional context. For this, we consider the exact solution (37) with $k=3$. The algorithms were given such exact Dirichlet data on each of the profiles $f=f_{s}, f_{r, P}, f_{L, P}(P=40)$ scaled by a particular value $\varepsilon_{0}$ and were made to compute the corresponding Neumann data through the evaluation of the Taylor series of $G(\varepsilon f)$ in $\varepsilon$ at $\varepsilon=\varepsilon_{0}$. The results with $N_{x}=256$ and $N_{y}=64$ are given in Figs. 1-3. These figures display relative mean-squared errors as a function of $n$, the number of terms retained in the Taylor series, for each of the profiles when $\varepsilon_{0}=0.3$. The effect of the ill-conditioning of the $\mathrm{OE}$ and FE formulas is evidenced here in the form of an explosive divergence of the series beyond a few terms whose onset is precipitated by a profile's roughness. Indeed, the figures show that as the profile is varied from smooth to rough to Lipschitz, the onset of divergence for the OE and FE methods changes from $n=9$ to 8 to 4 and from $n=20$ to 13 to 5 , respectively. In contrast, the results of TFE are consistently stable and only limited by the numerical resolution $\left(N_{y}=64\right)$. A further refinement of the discretization in the $x$ direction (to $N_{x}=512$ ), results in a loss

TABLE III

Significant Digits in Real Part of $a_{n, 1}$; (Truncated)

Rough Profile (2D, $\left.N_{x}=256\right)$

\begin{tabular}{rrrr}
\hline$n$ & TFE & FE & OE \\
\hline 2 & 11 & 15 & 15 \\
4 & 11 & 14 & 14 \\
6 & 11 & 14 & 14 \\
8 & 10 & 13 & 14 \\
10 & 10 & 12 & 13 \\
12 & 10 & 10 & 12 \\
14 & 10 & 8 & 10 \\
16 & 10 & 7 & 8 \\
18 & 10 & 5 & 7 \\
20 & 10 & 3 & 5 \\
22 & 10 & 1 & 3 \\
24 & 9 & -1 & 1 \\
\hline
\end{tabular}


TABLE IV

Significant Digits in Real Part of $a_{n, 1}$; (Truncated)

Lipschitz Profile (2D, $\left.N_{x}=512\right)$

\begin{tabular}{rrrr}
\hline$n$ & TFE & FE & OE \\
\hline 2 & 11 & 16 & 16 \\
4 & 10 & 12 & 16 \\
6 & 10 & 10 & 12 \\
8 & 10 & 7 & 10 \\
10 & 9 & 4 & 6 \\
12 & 9 & 1 & 3 \\
14 & 8 & -1 & 0 \\
\hline
\end{tabular}

of accuracy in the $\mathrm{OE}$ and $\mathrm{FE}$ results for both the smooth and rough profiles, indicating that conditioning errors overcome those that may arise from aliasing. For the Lipschitz profile, on the other hand, a discretization with $N_{x}=512$ actually produces slightly better results (for both $\mathrm{OE}$ and FE), but they again deteriorate at $N_{x}=1024$; see Figs. 4 (FE results) and 5 (OE results). For comparison, these figures also include the results of TFE with $N_{x}=256$ which demonstrate a further and unexpected advantage of TFE over the $\mathrm{OE}$ and FE implementations, as the latter appear to contain more substantial information at high wavenumbers in each term of the perturbation series. Indeed, these figures show that at a resolution of $N_{x}=256$, the calculation of terms beyond $n=5$ with FE and $\mathrm{OE}$ are significantly aliased, while that of TFE is not (as demonstrated by the higher resolution results of FE and OE).

As we said, the results above were chosen to exemplify the performance of the methods over a range of roughness and spatial discretizations. Of course, the size of the perturbations also plays an important role, as it determines the rate of convergence. As we have found,

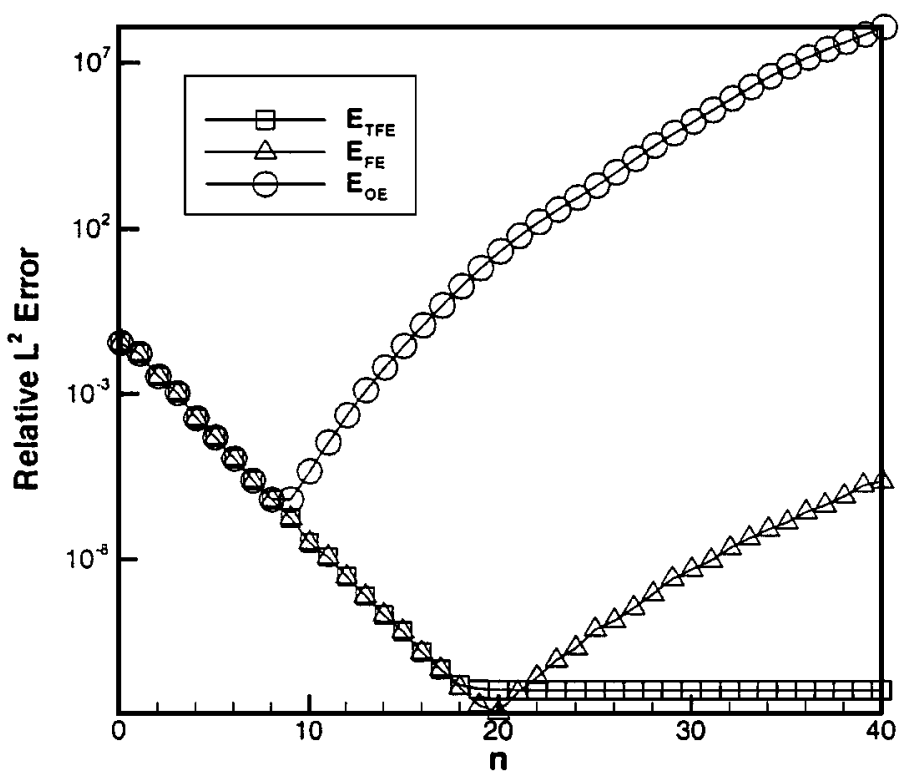

FIG. 1. Plot of relative $L^{2}$ errors versus $n$ : smooth profile in 2D with $\varepsilon_{0}=0.3, N_{x}=256$. 


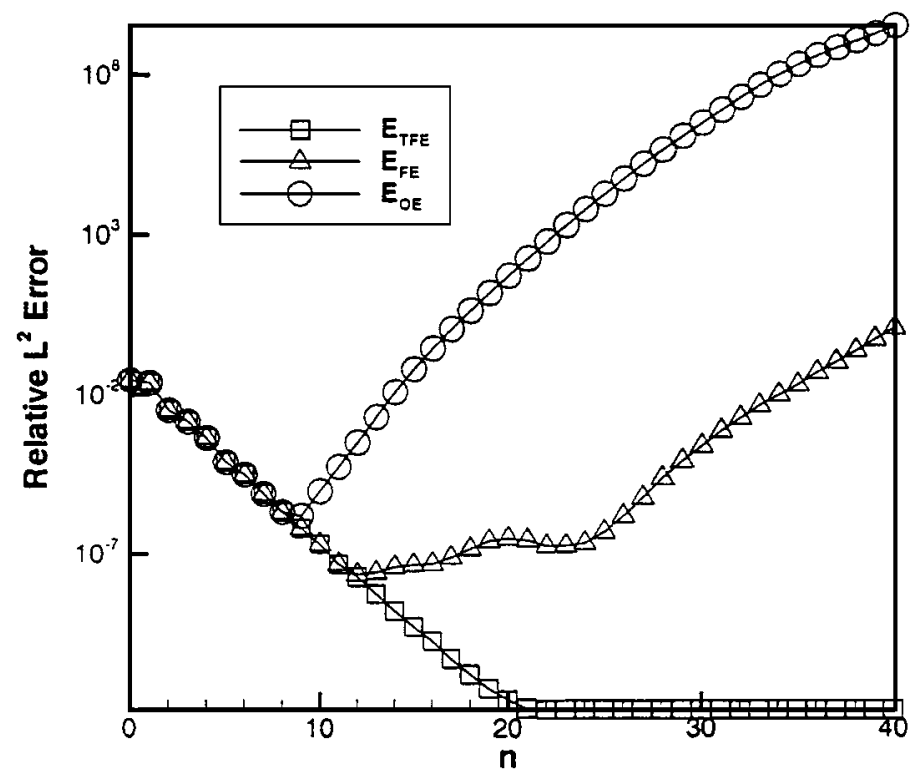

FIG. 2. Plot of relative $L^{2}$ errors versus $n$ : rough profile in $2 \mathrm{D}$ with $\varepsilon_{0}=0.3, N_{x}=256$.

the results we have presented are qualitatively general with regard to a change in the magnitude of the modulations. Indeed, convergence studies for other sizes reveal, as is to be expected, a change in the convergence rate but otherwise similar results for the number of terms $(n)$ that are accurately computed by each method. This, of course, implies that for larger perturbations the inability of FE or OE to accurately produce high-order terms may conspire against their resolution of the corresponding problem within an acceptable error.

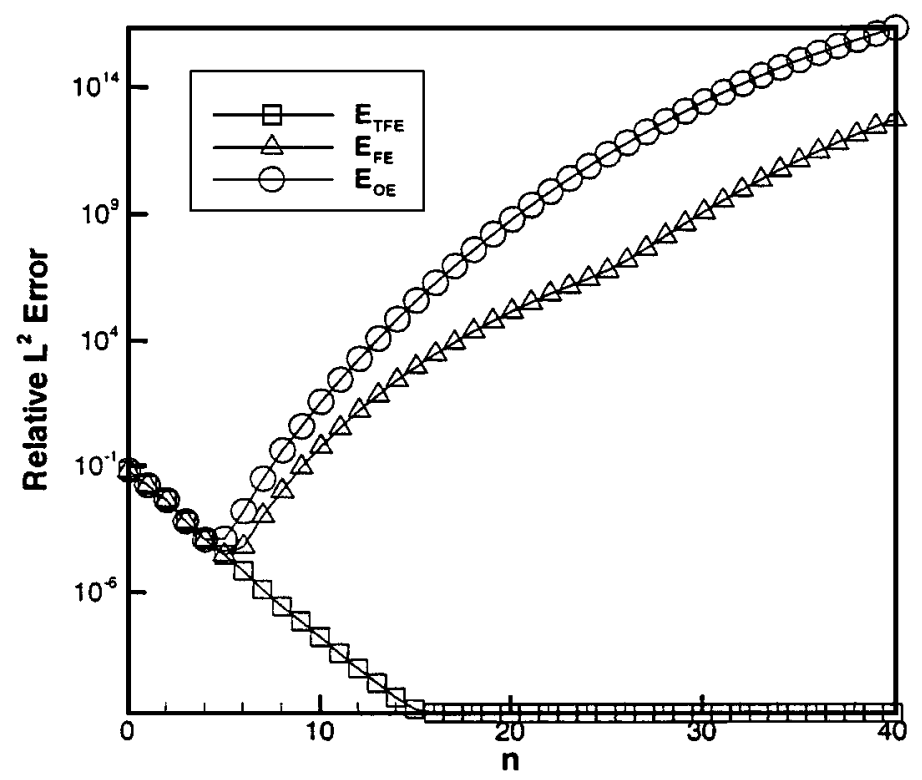

FIG. 3. Plot of relative $L^{2}$ errors versus $n$ : Lipschitz profile in $2 \mathrm{D}$ with $\varepsilon_{0}=0.3, N_{x}=256$. 


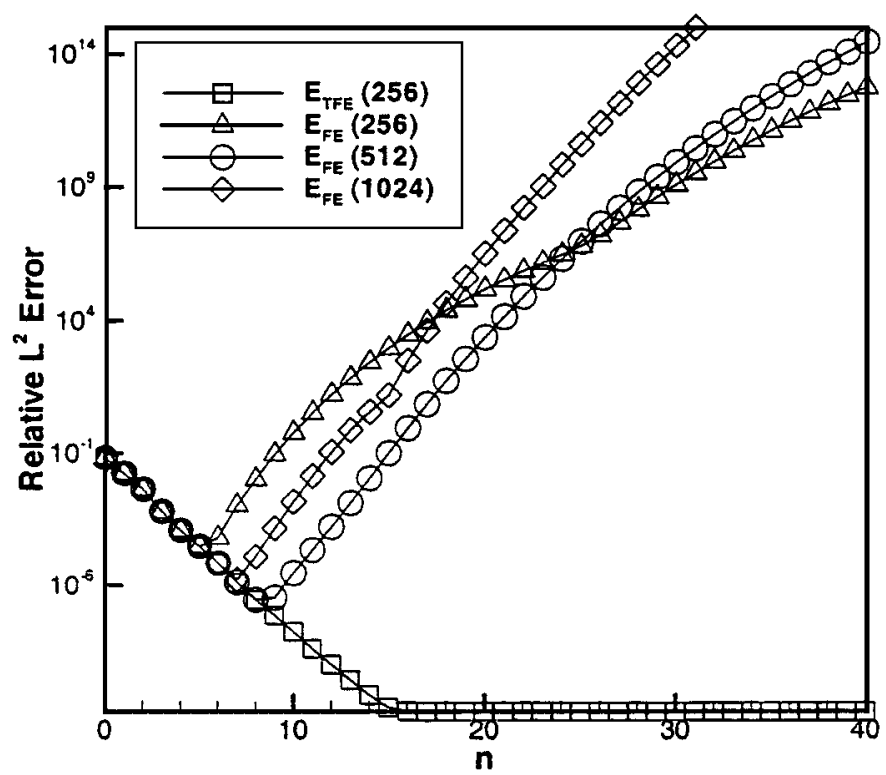

FIG. 4. Plot of relative $L^{2}$ errors in FE versus $n$ : Lipschitz profile in $2 \mathrm{D}$ with $\varepsilon_{0}=0.3, N_{x}=256,512$, and 1024.

To illustrate such a situation we display in Fig. 6 the results for a larger (rough) surface deformation $\left(\varepsilon_{0}=0.8\right)$, where a calculation with $n=9-13$ (the highest order terms that can be accurately computed with OE and FE) delivers an error of only $10^{-2}$.

4.3.2. Three-dimensional computations. In three dimensions we once again carried out illustrative computations based on three canonical geometries. On the domain $[0,2 \pi] \times$

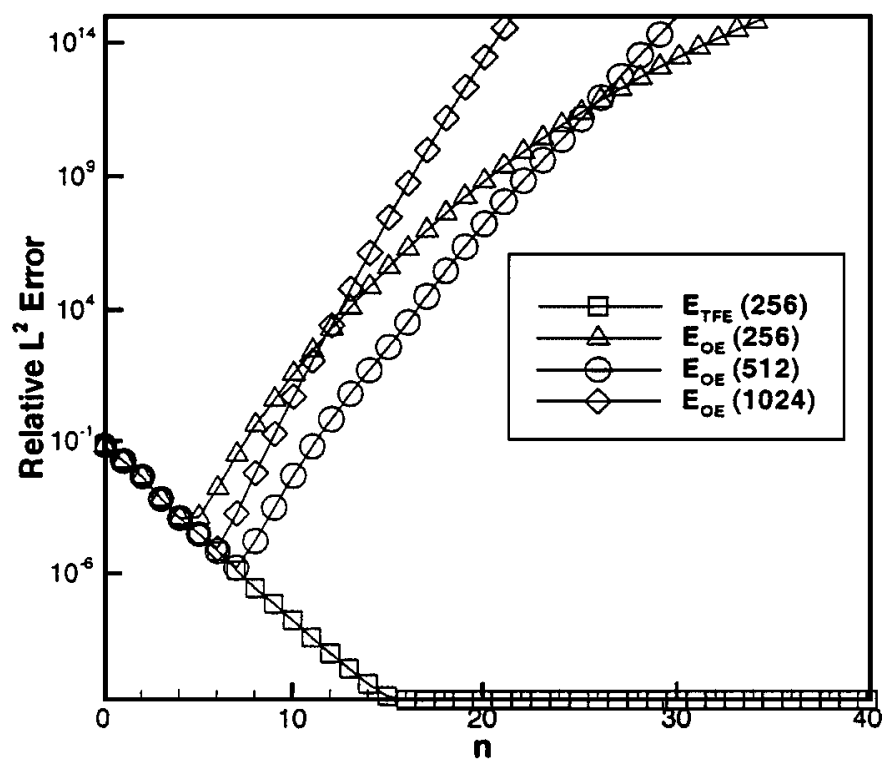

FIG. 5. Plot of relative $L^{2}$ errors in OE versus $n$ : Lipschitz profile in 2D with $\varepsilon_{0}=0.3, N_{x}=256,512$, and 1024. 


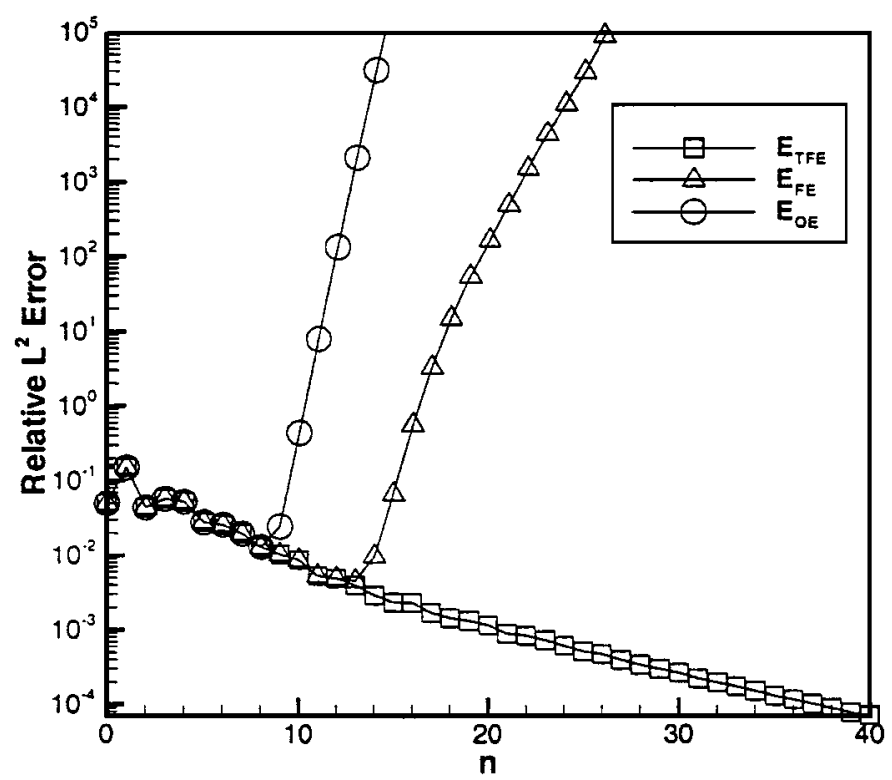

FIG. 6. Plot of relative $L^{2}$ errors versus $n$ : rough profile in $2 \mathrm{D}$ with $\varepsilon_{0}=0.8, N_{x}=256$.

$[0,2 \pi]$, we chose the smooth, rough (finite smoothness, $C^{2}$ ), and Lipschitz profiles

$$
\begin{aligned}
& g_{s}\left(x_{1}, x_{2}\right)=\cos \left(x_{1}+x_{2}\right) \\
& g_{r}\left(x_{1}, x_{2}\right)=\left(\frac{2}{9} \times 10^{-3}\right) x_{1}^{2}\left(2 \pi-x_{1}\right)^{2} x_{2}^{2}\left(2 \pi-x_{2}\right)^{2}-c_{1} \\
& g_{L}\left(x_{1}, x_{2}\right)= \begin{cases}-1+\frac{2}{\pi} x_{1} & \left\{\left(x_{1}, x_{2}\right) \in[0,2 \pi]^{2} \mid x_{1}<x_{2}<2 \pi-x_{1}\right\} \\
3-\frac{2}{\pi} x_{2} & \left\{\left(x_{1}, x_{2}\right) \in[0,2 \pi]^{2} \mid x_{2}>x_{1}, x_{2}>2 \pi-x_{1}\right\} \\
3-\frac{2}{\pi} x_{1} & \left\{\left(x_{1}, x_{2}\right) \in[0,2 \pi]^{2} \mid 2 \pi-x_{1}<x_{2}<x_{1}\right\} \\
-1+\frac{2}{\pi} x_{2} & \left\{\left(x_{1}, x_{2}\right) \in[0,2 \pi]^{2} \mid x_{2}<x_{1}, x_{2}<2 \pi-x_{1}\right\},\end{cases}
\end{aligned}
$$

where, as before, scalings were chosen so that maximum amplitudes and maximum slopes are approximately 2 and 1 , respectively, and $c_{1}$ so that $g_{r}$ has mean zero. The Fourier series representation of the two latter profiles are given by

$$
\begin{aligned}
g_{r}\left(x_{1}, x_{2}\right)= & \sum_{p=1}^{\infty} \sum_{k=1}^{\infty} \frac{32}{125 k^{4} p^{4}}\left[\cos \left(k x_{1}+p x_{2}\right)+\cos \left(k x_{1}-p x_{2}\right)\right] \\
& -\sum_{k=1}^{\infty} \frac{32 \pi^{4}}{5625 k^{4}} \cos \left(k x_{1}\right)-\sum_{p=1}^{\infty} \frac{32 \pi^{4}}{5625 p^{4}} \cos \left(p x_{2}\right) \\
g_{L}\left(x_{1}, x_{2}\right)= & -\frac{1}{3}+\sum_{k=1}^{\infty} \frac{2}{\pi^{2} k^{2}}\left[\cos \left(k\left(x_{1}+x_{2}\right)\right)\right. \\
& \left.+\cos \left(k\left(x_{1}-x_{2}\right)\right)-2 \cos \left(k x_{1}\right)-2 \cos \left(k x_{2}\right)\right] .
\end{aligned}
$$




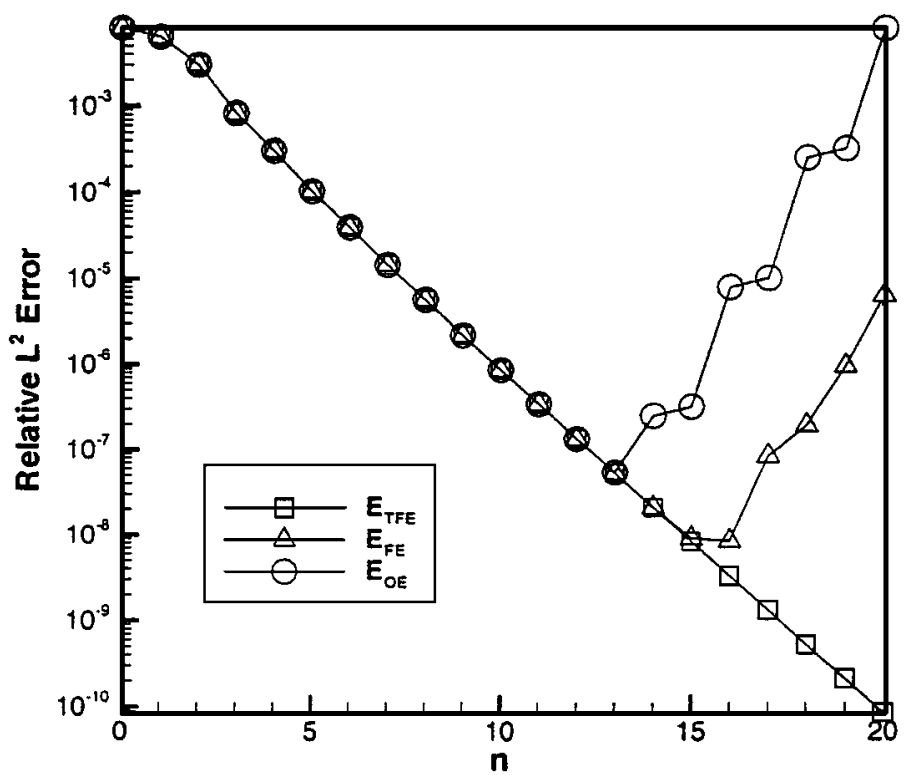

FIG. 7. Plot of relative $L^{2}$ errors versus $n$ : smooth profile in $3 \mathrm{D}$ with $\varepsilon_{0}=0.3, N_{x_{1}}=N_{x_{2}}=64$.

Again here, and in the interest of excluding aliasing errors, we approximated $g_{r}$ and $g_{L}$ by their truncated Fourier representation, $g_{r, P}$ and $g_{L, P}$ with $P=40$. In Figs. 7-9 we present the results of calculations corresponding to the normal derivative of the function $v_{3}\left(x_{1}, x_{2}, y\right)$ in Eq. (37) as computed with each method through the expansion of $G(\varepsilon g)$ evaluated at a specific value $\varepsilon=\varepsilon_{0}$. The figures show the relative $L^{2}$ errors in the Neumann data for $g_{s}, g_{r, P}$, and $g_{L, P}(P=40)$ for $\varepsilon_{0}=0.3, N_{y}=64$, and several values of $N_{x_{1}}$ and $N_{x_{2}}$. As in the two-dimensional case, the instability of the OE and FE procedures leads

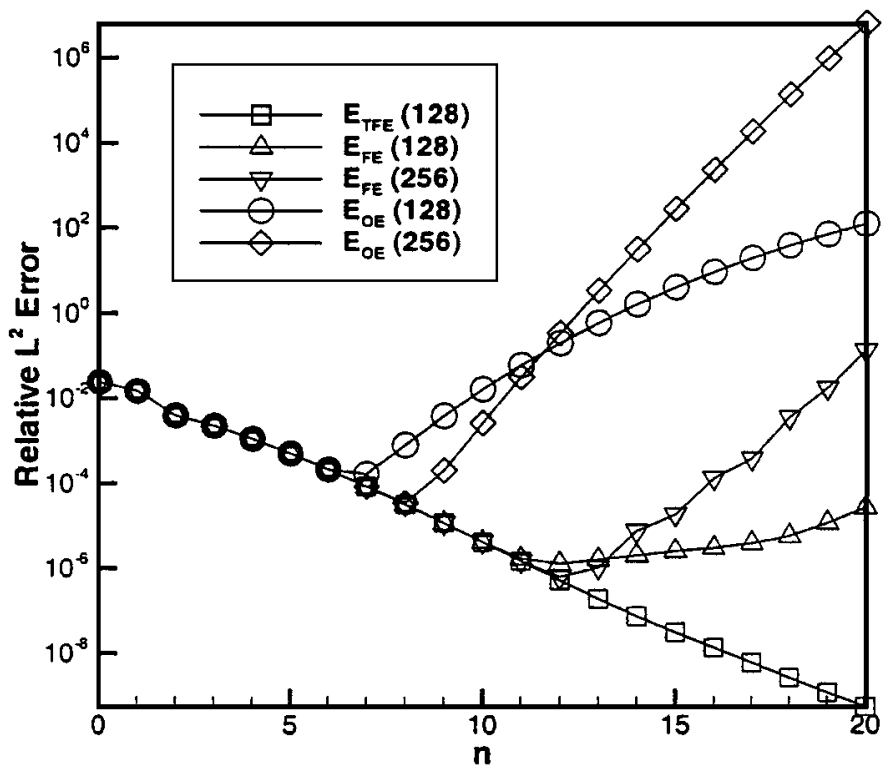

FIG. 8. Plot of relative $L^{2}$ errors versus $n$ : rough profile in $3 \mathrm{D}$ with $\varepsilon_{0}=0.3, N_{x_{1}}=N_{x_{2}}=128$ and 256 . 


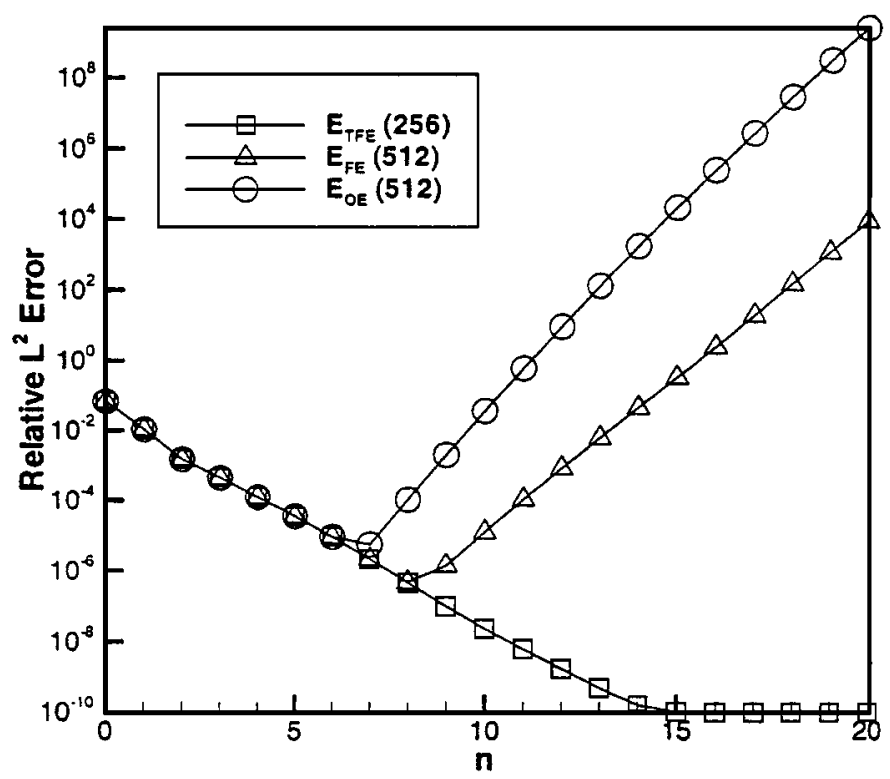

FIG. 9. Plot of relative $L^{2}$ errors versus $n$ : Lipschitz profile in $3 \mathrm{D}$ with $\varepsilon_{0}=0.3, N_{x_{1}}=N_{x_{2}}=512$.

to divergence at a critical value of the order $n$ of the Taylor expansion which depends on the smoothness of the underlying profile. For the smooth profile, Fig. 7 displays results corresponding to $N_{x_{1}}=N_{x_{2}}=64$ (and $N_{y}=64$, as in all cases); higher resolution in the horizontal variables leads to a loss of accuracy in the outcome of OE and FE. Figures 8 and 9 show the results for the rough and Lipschitz profiles, respectively. Here, however, results with $N_{x_{1}}=N_{x_{2}}=64$ are substantially underresolved (e.g., only three terms of the perturbation series can be accurately computed for the Lipschitz modulation). The figures show the results corresponding to the optimal values for the FE and OE implementations: $N_{x_{1}}=N_{x_{2}}=256$ for the rough surface and $N_{x_{1}}=N_{x_{2}}=512$ for the Lipschitz perturbation. Figure 8 also displays the results of all methods for a coarser discretization, $N_{x_{1}}=N_{x_{2}}=$ 128 , which again demonstrate the additional effect of the change of variables (25a) and (25b) of reducing the high-frequency content of higher order terms in the perturbation series (cf. Section 4.3.1). For the Lipschitz profile, in turn, the results in Fig. 9 show that a coarser TFE calculation $\left(N_{x_{1}}=N_{x_{2}}=256\right)$ suffices to resolve almost twice as many terms in the corresponding Taylor series as those that can possibly be computed with OE and FE and which, in this case, are no more than 6 and 8, respectively.

Finally, and as in the two-dimensional case, results for larger surface deformations are qualitatively similar, as the size only influences the rate of convergence (see Fig. 6). Thus, as anticipated, we conclude that an alternative approach, such as TFE, may be necessary in cases that demand the resolution of high-order harmonics (rough profiles) or higher order terms in the perturbation series (moderate to large perturbations).

\section{ACKNOWLEDGMENTS}

DPN thankfully acknowledges helpful discussions with Jan Hesthaven and Guido Kanschat. FR gratefully acknowledges support from AFOSR through Contract F49620-99-1-0193 and from NSF through Grant DMS-9971379. Effort sponsored by the Air Force Office of Scientific Research, Air Force Materials Command, 
USAF, under Grant F49620-99-1-0193. The U.S. Government is authorized to reproduce and distribute reprints for governmental purposes notwithstanding any copyright notation thereon. The views and conclusions contained herein are those of the authors and should not be interpreted as necessarily representing the official policies or endorsements, either expressed or implied, of the Air Force Office of Scientific Research or the U.S. Government.

\section{REFERENCES}

1. O. P. Bruno and F. Reitich, Solution of a boundary value problem for Helmholtz equation via variation of the boundary into the complex domain, Proc. Royal Soc. Edinburgh 122A, 317 (1992).

2. O. P. Bruno and F. Reitich, Numerical solution of diffraction problems: a method of variation of boundaries II. Dielectric gratings, Padé approximants and singularities, J. Opt. Soc. Amer. A 10, 2307 (1993).

3. Oscar P. Bruno and Fernando Reitich, Approximation of analytic functions: a method of enhanced convergence, Math. Comp. 63, 95 (1994).

4. A. P. Calderón, Cauchy integrals on Lipschitz curves and related operators, Proceedings of the National Academy Sciences USA 75, 324 (1977).

5. C. Canuto, M. Y. Hussaini, A. Quarteroni, and T. A. Zang, Spectral Methods in Fluid Mechanics (SpringerVerlag, New York, 1988).

6. R. Coifman, M. Goldberg, T. Hrycak, M. Israeli, and V. Rokhlin, An improved operator expansion algorithm for direct and inverse scattering computations, Waves Random Media 9, 71 (1999).

7. R. Coifman and Y. Meyer, Nonlinear harmonic analysis and analytic dependence, in Pseudodifferential Operators and Applications (Amer Math. Soc., Notre Dame, Ind., 1984), pp. 71-78.

8. W. Craig and D. P. Nicholls, Traveling two and three dimensional capillary gravity water waves, SIAM J. Math. Anal. 32(2), 323 (2000).

9. W. Craig and C. Sulem, Numerical simulation of gravity waves, J. Comput. Phys. 108, 73 (1993).

10. W. Craig, U. Schanz, and C. Sulem, The modulation regime of three-dimensional water waves and the DaveyStewartson system, Ann. Inst. Henri Poincaré 14, 615 (1997).

11. D. G. Dommermuth and D. K. P. Yue, A high-order spectral method for the study of nonlinear gravity waves, J. Fluid Mech. 184, 267 (1987).

12. D. Givoli, Exact representations on artificial interfaces and applications in mechanics, Appl. Mech. Rev. 52, 333 (1999).

13. M. J. Grote, Nonreflecting boundary conditions for elastodynamic scattering, J. Comput. Phys. 161, 331 (2000).

14. D. Gottlieb and S. A. Orszag, Numerical Analysis of Spectral Methods: Theory and Applications, (Society for Industrial and Applied Mathematics, Philadelphia, PA, 1977), CBMS-NSF Regional Conference Series in Applied Mathematics, No. 26.

15. H. Hasimoto and H. Ono, Nonlinear modulation of gravity waves, J. Phys. Soc. Japan 33(3), 805 (1972).

16. V. Isakov, Inverse problems for partial differential equations, in Applied Mathematical Sciences, 127 (SpringerVerlag, New York, 1998).

17. P. J. Kaczkowski and E. I. Thorsos, Application of the operator expansion method to scattering from onedimensional moderately rough Dirichlet random surfaces, J. Acoust. Soc. Am. 96(2), 957 (1994).

18. D. M. Milder, The effects of truncation on surface-wave Hamiltonians, J. Fluid Mech. 216, 249 (1990).

19. D. M. Milder, An improved formalism for rough-surface scattering of acoustic and electromagnetic waves, in Proceedings of SPIE-The International Society for Optical Engineering, San Diego, 1991 (Int. Soc. for Optical Engineering, Bellingham, WA, 1991), Vol. 1558, pp. 213-221.

20. D. M. Milder, An improved formalism for wave scattering from rough surfaces, J. Acoust. Soc. Am. 89(2), 529 (1991).

21. D. M. Milder, An improved formalism for electromagnetic scattering from a perfectly conducting rough surface, Radio Sci. 31(6), 1369 (1996).

22. D. M. Milder, Role of the admittance operator in rough-surface scattering, J. Acoust. Soc. Am. 100(2), 759, (1996). 
23. D. M. Milder and H. T. Sharp. Efficient computation of rough surface scattering, in Mathematical and Numerical Aspects of Wave Propagation Phenomena (Strasbourg, 1991), (SIAM, Philadelphia, PA, 1991), pp. 314-322.

24. D. M. Milder and H. T. Sharp, An improved formalism for rough surface scattering. ii: Numerical trials in three dimensions, J. Acoust. Soc. Am. 91(5), 2620 (1992).

25. E. H. Mund, A short survey of preconditioning techniques in spectral calculations, Appl. Numer. Math. 33, 61 (2000).

26. D. P. Nicholls and F. Reitich, A new approach to analyticity of Dirichlet-Neumann operators, submitted for publication.

27. Ralph A. Smith, An operator expansion formalism for nonlinear surface waves over variable depth, J. Fluid Mech. 363, 333 (1998).

28. Gunther Uhlmann, Developments in inverse problems since Calderón's foundational paper, in Harmonic Analysis and Partial Differential Equations Chicago Lectures in Mathematics (Univ. Chicago Press, Chicago, IL, 1999) pp. 295-345.

29. Alexander G. Voronovich, Wave Scattering from Rough Surfaces, 2nd ed. (Springer-Verlag, Berlin, 1999).

30. K. M. Watson and B. J. West, A transport-equation description of nonlinear ocean surface wave interactions, J. Fluid Mech. 70, 815 (1975). 